\title{
Sleep Disorders During Pregnancy and Postpartum: A Systematic Review
}

\author{
Zahra Behboodi Moghadam, $\mathrm{PhD}^{1}$, Elham Rezaei, $\mathrm{PhD}^{2}$, Azam Rahmani, $\mathrm{PhD}^{3}$ \\ ${ }^{1}$ Reproductive Health, School of Nursing and Midwifery, Tehran University of Medical Sciences, Tehran, Iran \\ ${ }^{2}$ Reproductive Health, Reproductive Health Research Center, Clinical Research Institute, Urmia University of Medical Sciences, Urmia, Iran \\ ${ }^{3}$ Reproductive Health, Nursing and Midwifery Care Research Center, School of Nursing and Midwifery, Tehran University of Medical Sciences, Tehran, Iran
}

Received: July 3, 2021

Revised: September 22, 2021

Accepted: November 2, 2021

Correspondence

Elham Rezaei, $\mathrm{PhD}$

Reproductive Health,

Reproductive Health Research Center,

Clinical Research Institute,

Urmia University of Medical Sciences,

Orjhans Street, Resalat Blvd,

Urmia 571478334, Iran

Tel +989144633116

Fax +04433469935

E-mail rezai520@yahoo.com

ORCID

Zahra Behboodi Moghadam

https://orcid.org/0000-0003-2889-802X

Elham Rezaei

https://orcid.org/0000-0002-5461-5903

Azam Rahmani

https://orcid.org/0000-0001-8528-2374

(C) This is an Open Access article distributed under the terms of the Creative Commons Attribution Non-Commercial License (https://creativecommons.org/licenses/by-nc/4.0) which permits unrestricted non-commercial use, distribution, and reproduction in any medium, provided the original work is properly cited.
This study aimed to asses previous research results about bio-physiological alterations during pregnancy and postpartum, and make clear outlook about prevalence and related factors of sleep disorders during pregnancy and postpartum. In this review, the articles that published from 2000 to 2019 were reviewed. Related articles were searched from databases in English language. After evaluation of inclusion and exclusion criteria, articles were chosen and reviewed based on the University of York strategies. Sleep disorders in present study were classified according to the International Classification of Sleep Disorders-3. The search revealed 4449 articles, after evaluating and assessing qualified articles, finally 56 article selected to review. According to the results of this review, The prevalence of sleep disorders was almost $76 \%-97 \%$ in whole pregnancy. The most common sleep disorders included central disorders of hyper somnolence (waking up in the middle of the night, daytime sleepiness, sleeplessness) or insomnia, sleep-related movement disorders (restless legs syndrome), sleep related breathing disorders (obstructive sleep apnea), and parasomnia. In addition, sleep disorder may continue 3-12 months postpartum (33.2\%). Sleep disorders induced by physiological processes (fetal movement, excessive weight gain, male sex of the fetus, and multiparty), health-related risks (metabolism disorders, cardiovascular diseases, and mood disorders), and physical or sexual abuse in childhood. In addition, these disorders could have maternal outcomes that can be greatest trigger to postpartum psychiatric disorders and fetal outcomes that have harmful sequences during childhood (sexual, fertility, emotional, and cognitive problems). Health care providers should evaluate the mothers' sleep quality because sleep disorder leads to harmful consequences in fetuses and children.

Sleep Med Res 2021;12(2):81-93

Keywords Sleep disorders; Pregnancy; Postpartum; Systematic review.

\section{INTRODUCTION}

Sleep consists of non-rapid eye movement (NREM) sleep, also known as slow sleep, and rapid eye movement (REM) sleep or rapid sleep. REM sleep has an important role in memory reinforcement, organization of cognition ability, and mood regulation. NREM sleep is identified as a decreasing in physiological activity. It is believed that REM sleep which usually make up $20 \%-25 \%$ of the night sleep is important for cognitive functions [1].

Neonates need about 14 to 18 hours of sleep during the day which gradually decrease and reach 8 hours in adults. Sleep has a repairing and relieving function and is essential for preparing mothers to transfer their energy to fetal-placental unit [2]. So sleep disorders can interfere with these goals. Also, some studies emphasize the most prevalent sleep disorders are in first trimester of pregnancy vice versa other studies demonstrate in the second and third trimester [3-5]. As well as the prevalence of sleep disorder widely is deferent in the studies, Effati-Daryani et al. [6] demonstrated the prevalence of sleep disorder in the first (40.1\%), second (53.0\%), third (83.9\%), and whole pregnancy (59.2\%). But Jahdi et al. [7] showed the prevalence of sleep dis- 
order in the second or third trimesters $87.2 \%$. Hedman et al. [8] emphasized sleep disorder reduced during pregnancy. The limitation of evaluated studies was that most of these studies were cross-sectional which limited the assessment of real relationship between pregnancy situation and sleep disorders. Therefore, we decided to include qualified and reviewed articles in the present study to diminish this defeat. We planned to find the most prevalent sleep disorders during pregnancy, postpartum, and the mutual effects between sleep and pregnancy we hope to answer and confirm the contradictory results and make clear outlook about finding variations of different studies about pregnancy sleep disorders, their related factors, and recommend new insights to the future research.

\section{METHODS}

The articles published from 2000 to 2019 were reviewed according to the University of York strategies (Center for Reviews and Dissemination, University of York) [9]. We used University of York strategies because it includes all criteria of Cochran and is look like Cochran review as well as our authors use from this strategy frequently in their published articles.

Related articles were searched from the databases of Medline, Embase, Psych INFO, CINAHL, PubMed, OVID, EBSCO host, Science Direct, ISI Web of Knowledge, and Google Scholar. The search strategies were Pregnant Women; Pregnancy; Pregnancy Trimester (First, Second, Third); Postpartum Period; Postnatal Care; Sleep Wake Disorders; Dyssomnias; Sleep Disorders, Circadian Rhythm; Parasomnias; Restless Legs Syndrome (RLS); Sleep Arousal Disorders (Supplementary Table 1 in the onlineonly Data Supplement).

In addition, we applied some criteria to include relevant articles such as 1) Document type: article; 2) Language: English; 3) Study design: quantitative and qualitative; and 4) Existing essential keywords in title or abstract.

Two authors independently searched the databases and then screened the titles and abstracts of articles. Afterward, two authors independently studied full texts of the articles that met the criteria. Every disagreement was negotiated between two authors to acquire consensus and inter-ratter reliability. To add the richness of the review, we used both qualitative and quantitative articles in our research.

Furthermore, we used the Strengthening the Reporting of Observation Studies in Epidemiology (STROBE) which consists 22 items to assess cross-sectional, case-control, and cohort studies; Consolidated Standards of Reporting Trials (CONSORT) with 25 items to evaluate clinical trial studies and Preferred Reporting Items for Systematic Reviews and Meta-Analyses (PRISMA) tools consists 27 items to assess quality of review articles. We evaluated the methodology, managing the risk of biases, type and manner of analysis, evaluation of outcomes and de- fects in the discussion and research field. Then try to integrated quantitative and qualitative results. To integrate quantitative and qualitative data, we reported the results of the percentage of the most common sleep disorders in quantitative studies that had been reported one of the most common complaints and main concerns of women in qualitative studies.

Generally, we found 4449 articles, 3799 abstract and title were evaluated. 3019 articles were excluded because repeated researches $(n=650)$, irrelevant articles according to study goals (weren't on the pregnancy and postpartum) $(\mathrm{n}=950)$ and non-English papers ( $\mathrm{n}=59$ ), and unavailable full texts to make assessment $(n=200)$. Then quality of 580 articles was assessed by checklists. 523 articles excluded again because no actual data available on outcome $(n=109)$, outcomes not reported clearly $(n=80)$, not appropriate design/method $(n=170)$, background $(n=80)$, discussion $(\mathrm{n}=142)$. Finally 56 articles selected to review (Fig. 1 and Table 1). We used SPSS version 23.0 (IBM Corp., Armonk, NY, USA for analyzing data. Categorical variables were stated as number (\%) and continuous variables were represented as ranges.

\section{RESULTS}

Overall, 56 articles of different countries evaluated based on the mentioned searching methods were as follows: (Table 1): $14(26 \%)$ in the USA, 8 (15\%) in Iran, 5 (8.7\%) in Finland, 4 (7\%) in China, 4 (7\%) in Australia, 3 (5.2) in Taiwan, 2 (3.5\%) in Peru, 2 (3.5\%) in the UK, 2 (3.5\%) in Canada, 2 (3.5\%) in Turkey, 2 (3.5\%) in India, 1 (1.7\%) in Norway, 1 (1.7\%) in Brazil, 1 (1.7\%) in France, 1 (1.7\%) in New Zealand, 1 (1.7\%) in Sri Lanka, 1 (1.7\%) in Slovakia, 1 (1.7\%) in Russia, and 1 (1.7\%) in Spain.

All types of studies quantitative such as cross-sectional, casecontrol randomized controlled trials, retrospective and prospective cohort studies, reviews and content analysis were included. The findings of these 56 articles have been presented in the following section (Table 1 and Fig. 1). Also quality assessment of the articles have been showed in Table 2 .

\section{Effect of Pregnancy on Sleep}

Pregnancy affects women's sleep through making changes in structure and function of the body, hormone level, and immune system which lead to neonatal development and safe birth [10]. Most complaints start at the end of pregnancy and continue until a year after labor. Lee et al. [1] believed that changes in sleep pattern started from the end of the first trimester (11-12 weeks) of pregnancy; these changes included increased duration and wakefulness of sleep and decreased deep sleep that resulted in reducing sleep efficiency started from the follicular stage sometimes. Despite sleep improvement three months after labor, sleep is still less efficient than before. The sleep of multiparous women is less efficient before and after pregnancy, and they experience 


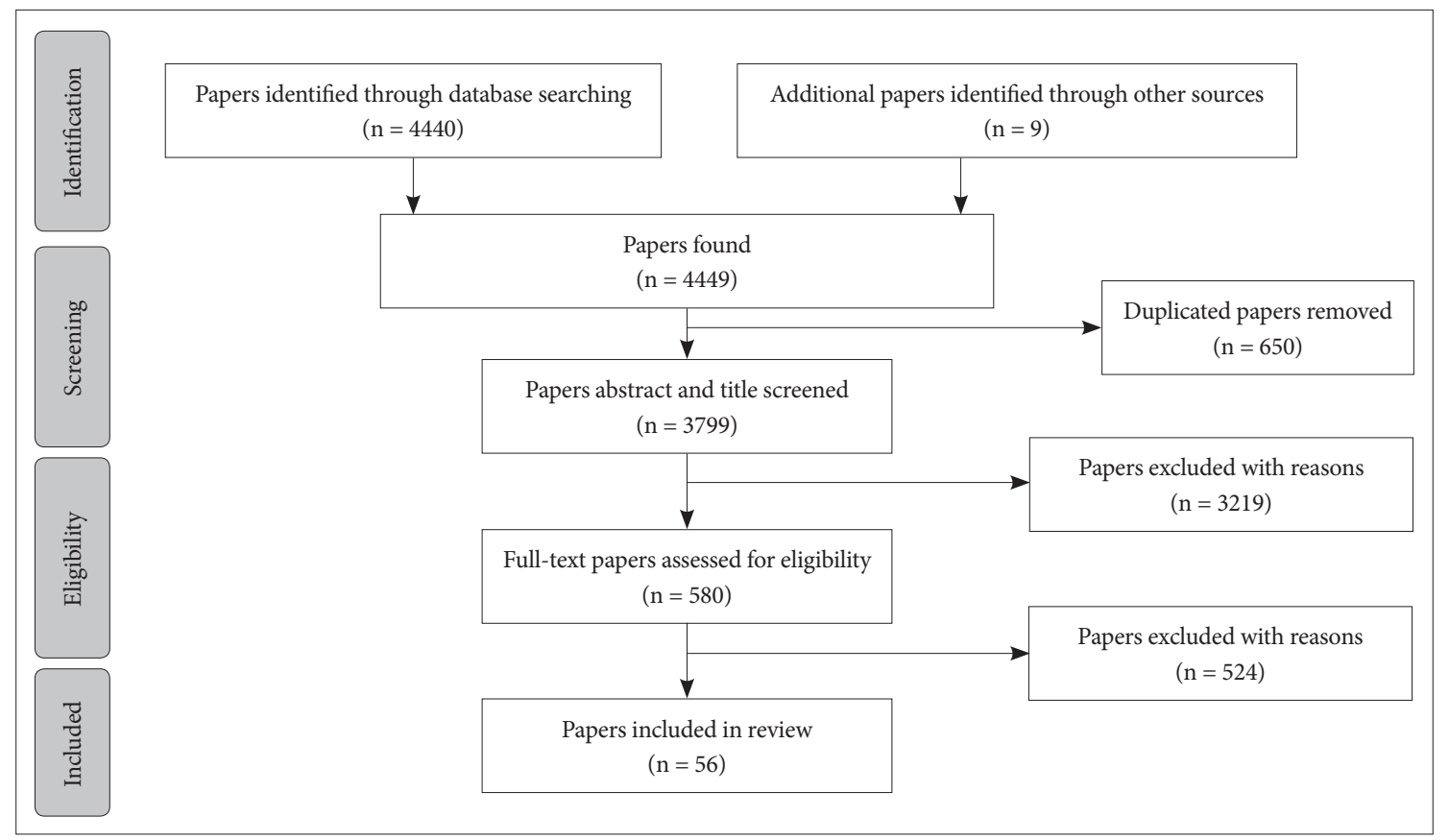

Fig. 1. Flow chart of the study's selection process based on Preferred Reporting Items for Systematic Reviews and Meta-Analyses (PRISMA).

repeated wakefulness more than nulliparous women [1].

Pregnancy imposes some additional needs such as more sleeping time. An increase in sleep causes mothers to store energy, which is useful for fetal nutrition and organogenesis. Increase in sleep duration increases human chorionic gonadotropin (HCG) and progesterone hormones that are essential for pregnancy continuation. Sleep duration increases in the first and second trimesters, but it decreases in the third trimester because of physiological changes $[3,5]$.

Furthermore, progesterone, estrogen, cortisol, and oxytocin increase gradually during pregnancy and affect sleep quality remarkably, especially in the third trimester. High progesterone in the first trimester causes sleepiness. High oxytocin can result in repeated waking-ups. Li et al. [11] demonstrated that overall sleep quality was weak at the end of pregnancy, and sleeping problems, night diseases, and early morning wake ups increased in comparison with the mid-pregnancy. Sleep quality and healthy life decrease remarkably in the second and third trimesters compared with the first trimester $[3,5]$.

Additionally, excessive daily sleeplessness has been observed in the first trimester more. Sleeplessness rate and repeated waking ups in women have increased during the second and third trimesters and reached 75\% especially because of an urgent need for urination, fetal movements, backache, and RLS [4]. Stomachache (a common physical symptom in pregnancy), nausea, polyuria, and backache may disturb sleep quality [2,12], as $38.8 \%$ of the pregnant women have poor sleep quality because of nausea and moderate or severe vomiting [11]. The fetal activity is effective in most mothers' waking ups during sleep in the third trimester [7]. After labor, neonates' nutritional requirements and subsequently repeated waking ups lead to sleep deprivation, stress, and different sleep disorders such as sleep respiratory distress, sleeplessness, and sleep attack. Although poor sleep, sleeplessness, and insufficient sleep are almost common in pregnant women, all women are not affected by sleep disorders $[11,13]$.

Results of Li et al. [11] demonstrated that the more flexible in facing problems, tragedies, and stress sources, the better the sleep quality. People who are more flexible have positive characteristics (such as high self-efficiency, positive feeling, realistic optimism, and cognitive flexibility), which help them for positive adaptation and preservation of good sleep quality against acute or chronic stress [11]. In addition, insufficient sleep is a known stress factor for health and a risk factor for pregnant women's health and fetal growth. In studies, $25 \%$ of women reported a high level of the labor fear (a complex picture of women's emotional experiences during pregnancy) and 20.6\% reported less than 6 hours of sleep each night. Women who are highly scared of labor have more daily stress, anxiety, fatigue, and less support, which cause about $95 \%$ of repeated night wake ups, $14 \%$ of sleep start problems, and $27 \%$ of low sleep quality $[13,14]$. On the other hand, more than $75 \%$ of the women had reported reduced conscious and increased need for day naps [4].

Studies have mentioned a relationship between short sleep duration (SSD), severe sleep disorder and high body mass index (BMI) in pregnant adults. Gay et al. [15] demonstrated that excessive weight gain was associated with shorter sleep duration and more sleep disorders. Moreover, Abeysena and Jayawardana [16] reported that daily sleep duration less than 8 hours in the second, third, or both second and third trimesters (odds ratio $[\mathrm{OR}]=1.60)$, male sex of fetus $(\mathrm{OR}=1.50)$, several pregnan- 


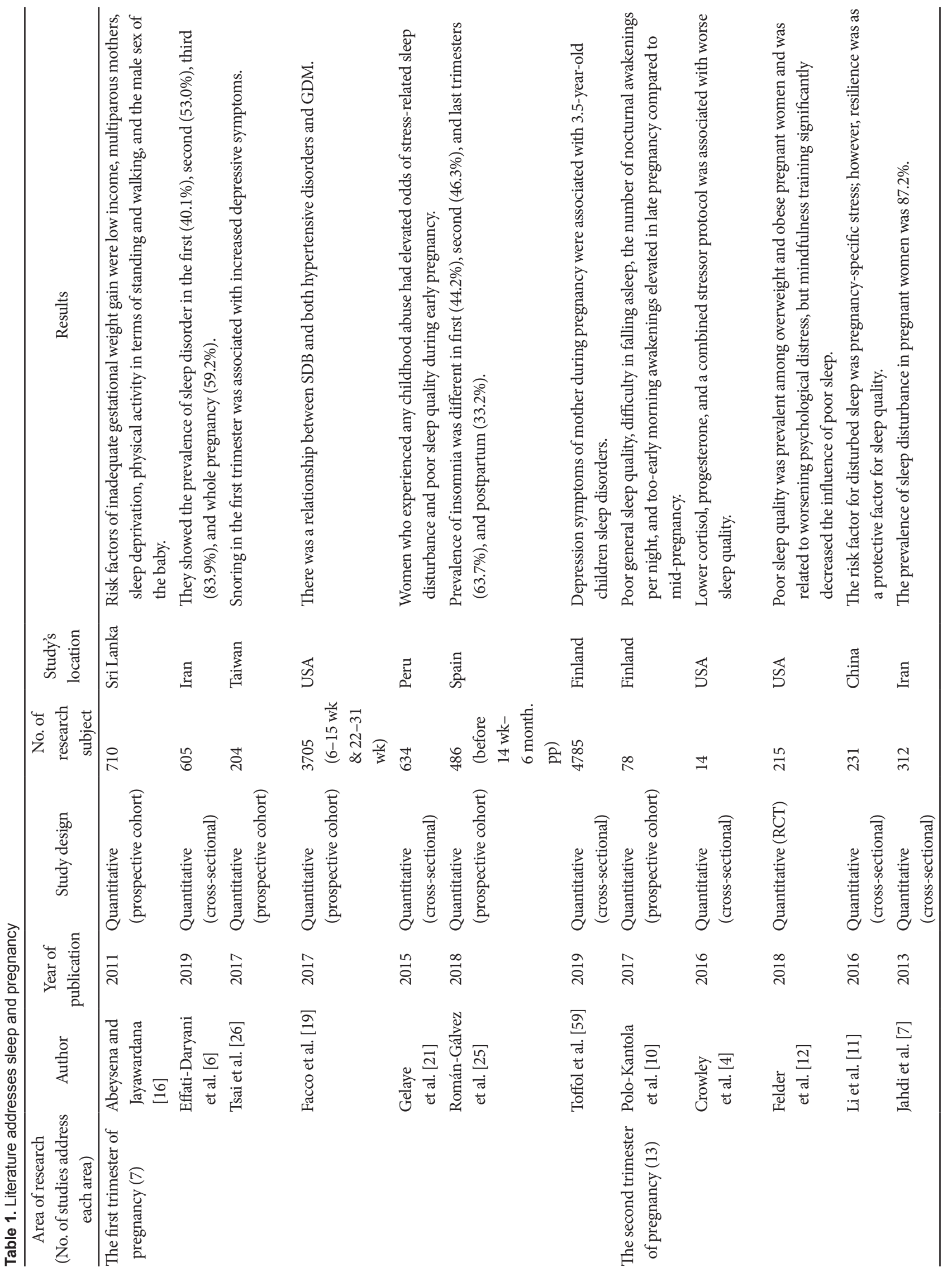




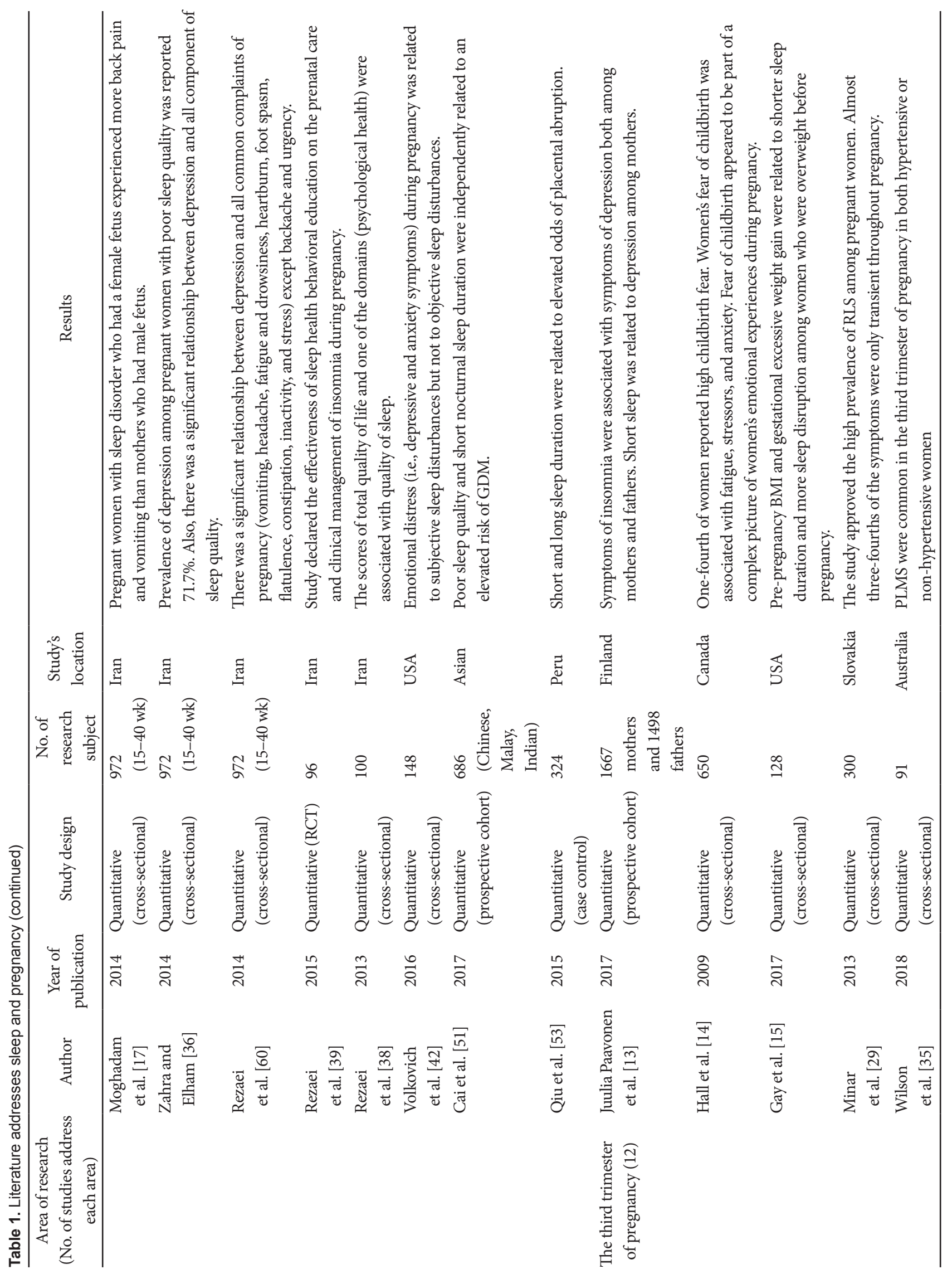




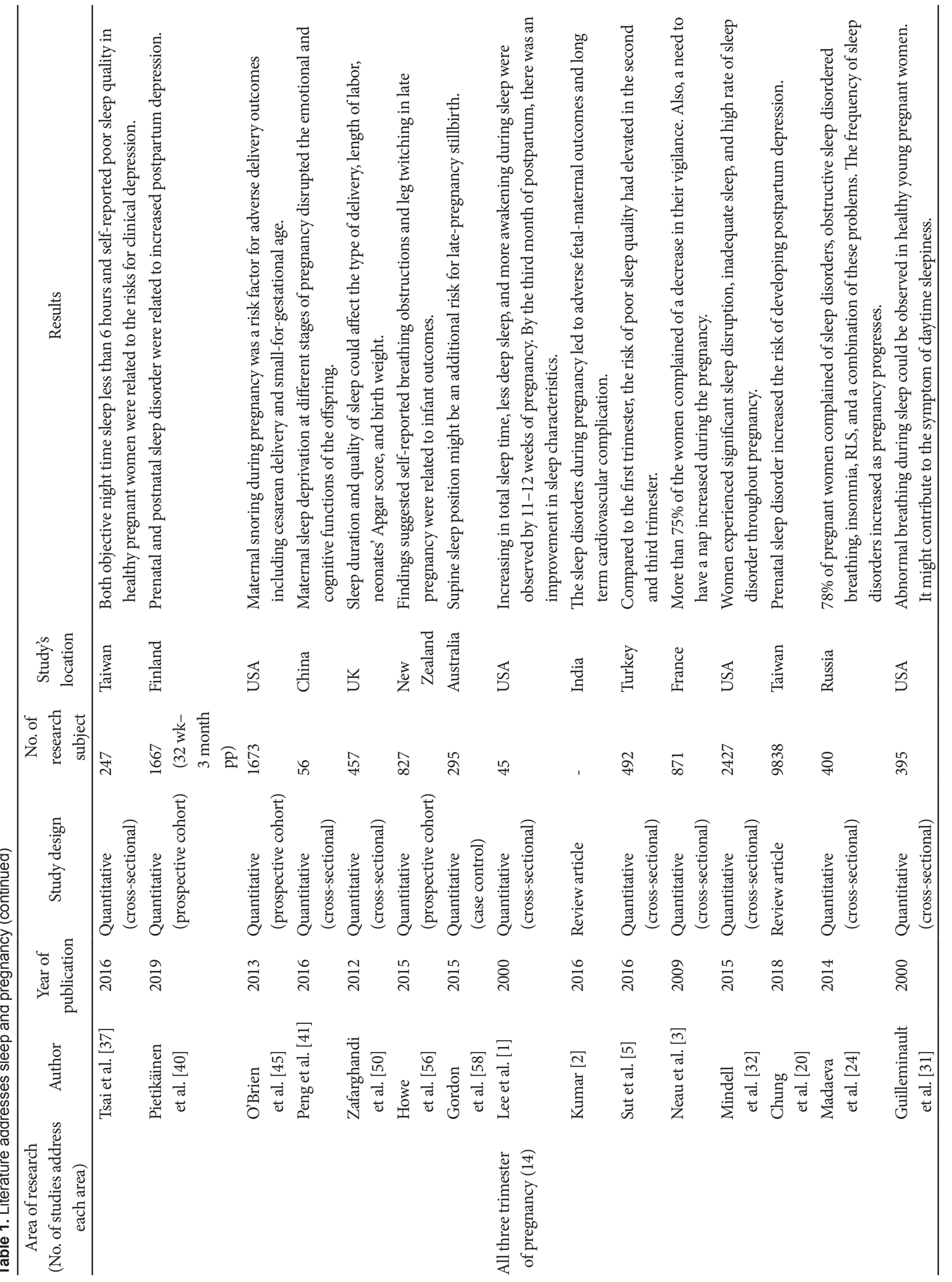




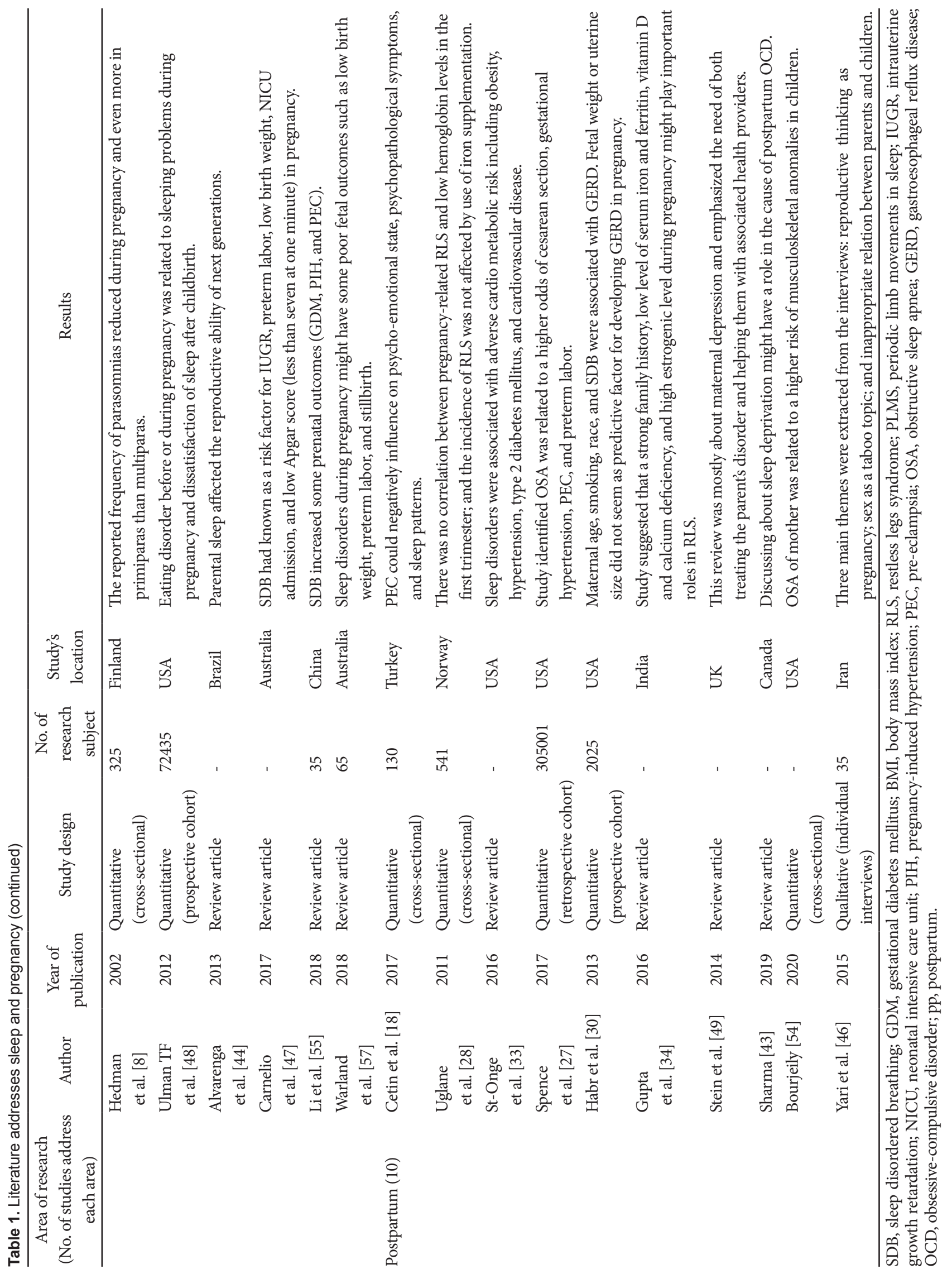


Table 2. Quality assessment of included articles by special check lists

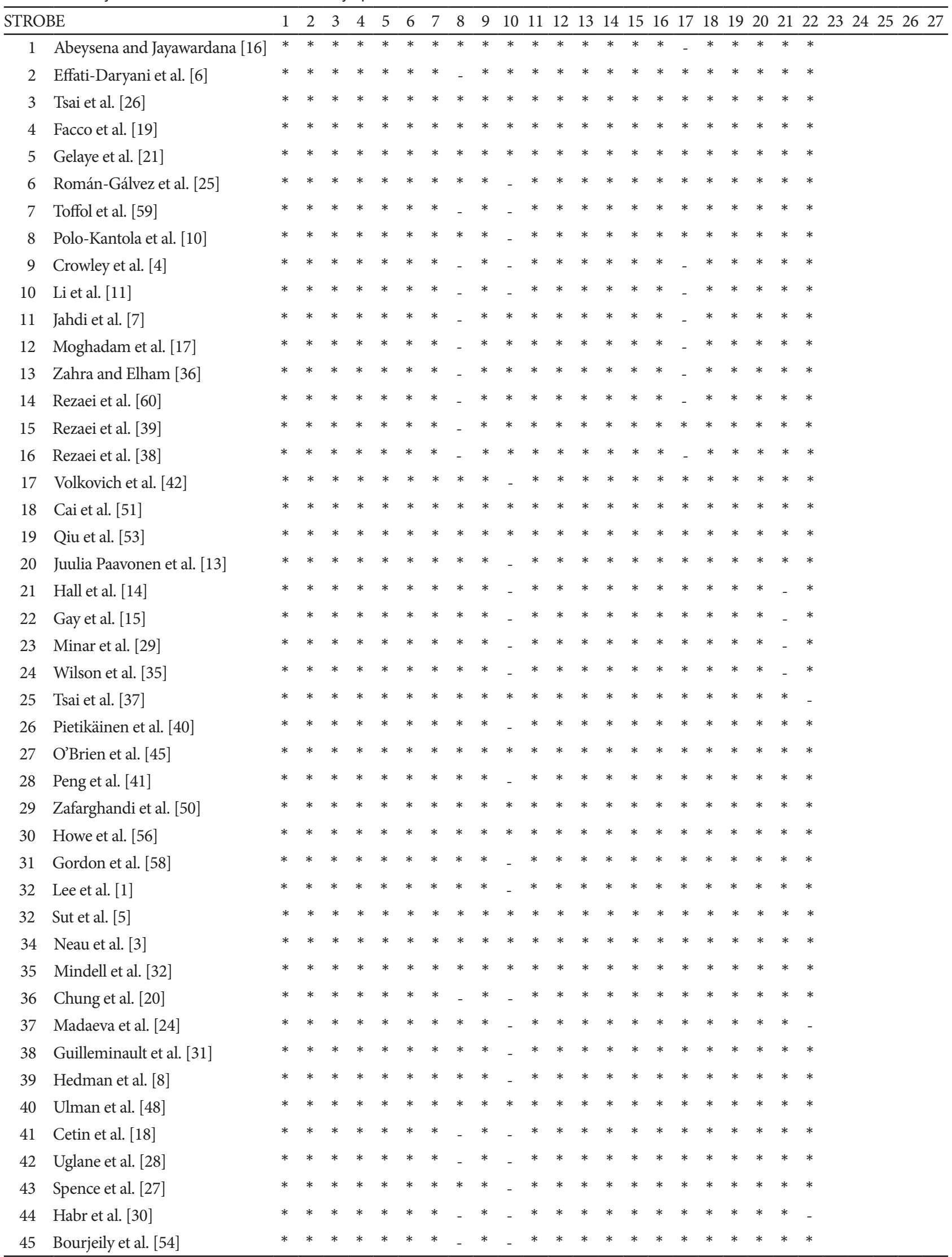


Table 2. Quality assessment of included articles by special check lists (continued)

\begin{tabular}{lllllllllllllllllllllllllllll}
\hline PRISMA & 1 & 2 & 3 & 4 & 5 & 6 & 7 & 8 & 9 & 10 & 11 & 12 & 13 & 14 & 15 & 16 & 17 & 18 & 19 & 20 & 21 & 22 & 23 & 24 & 25 & 26 & 27 \\
\hline 46 & Kumar [2] & $*$ & $*$ & $*$ & $*$ & - & $*$ & $*$ & $*$ & - & - & - & $*$ & $*$ & $*$ & - & - & $*$ & $*$ & $*$ & $*$ & $*$ & $*$ & $*$ & $*$ & $*$ & $*$ & $*$ \\
47 & Alvarenga et al. [44] & $*$ & $*$ & $*$ & $*$ & $*$ & $*$ & $*$ & $*$ & $*$ & $*$ & $*$ & $*$ & $*$ & $*$ & $*$ & $*$ & $*$ & $*$ & $*$ & $*$ & $*$ & $*$ & $*$ & $*$ & $*$ & $*$ & $*$ \\
48 & Carnelio et al. [47] & $*$ & $*$ & $*$ & $*$ & - & $*$ & $*$ & $*$ & - & $*$ & - & $*$ & $*$ & $*$ & $*$ & $*$ & $*$ & $*$ & $*$ & $*$ & $*$ & $*$ & $*$ & $*$ & $*$ & $*$ & $*$ \\
49 & Li et al. [55] & $*$ & $*$ & $*$ & $*$ & $*$ & $*$ & $*$ & $*$ & $*$ & $*$ & $*$ & $*$ & $*$ & $*$ & $*$ & $*$ & $*$ & $*$ & $*$ & $*$ & $*$ & $*$ & $*$ & $*$ & $*$ & $*$ & $*$ \\
50 & Warland et al. [57] & $*$ & $*$ & $*$ & $*$ & $*$ & $*$ & $*$ & $*$ & $*$ & $*$ & $*$ & $*$ & $*$ & $*$ & $*$ & $*$ & $*$ & $*$ & $*$ & $*$ & $*$ & $*$ & $*$ & $*$ & $*$ & $*$ & $*$ \\
51 & St-Onge et al. [33] & $*$ & $*$ & $*$ & $*$ & - & $*$ & $*$ & $*$ & - & - & $*$ & $*$ & $*$ & $*$ & - & $*$ & $*$ & $*$ & $*$ & $*$ & $*$ & $*$ & $*$ & $*$ & $*$ & $*$ & $*$ \\
52 & Gupta et al. [34] & $*$ & $*$ & $*$ & $*$ & - & $*$ & $*$ & $*$ & - & - & - & $*$ & $*$ & $*$ & - & $*$ & $*$ & $*$ & $*$ & $*$ & $*$ & $*$ & $*$ & $*$ & $*$ & $*$ & $*$ \\
53 & Stein et al. [49] & $*$ & $*$ & $*$ & $*$ & - & $*$ & $*$ & $*$ & $*$ & - & - & $*$ & $*$ & $*$ & - & $*$ & $*$ & $*$ & $*$ & $*$ & $*$ & $*$ & $*$ & $*$ & $*$ & $*$ & $*$ \\
54 & Sharma [43] & $*$ & - & $*$ & $*$ & - & $*$ & $*$ & $*$ & $*$ & - & - & $*$ & $*$ & $*$ & - & - & $*$ & $*$ & $*$ & $*$ & $*$ & $*$ & $*$ & $*$ & $*$ & $*$ & $*$ \\
CONSORT & & & & & & & & & & & & & & & & & & & & & & & & & & & \\
55 & Felder et al. [12] & $*$ & $*$ & $*$ & $*$ & $*$ & $*$ & $*$ & $*$ & $*$ & $*$ & $*$ & $*$ & $*$ & $*$ & $*$ & $*$ & $*$ & $*$ & $*$ & $*$ & $*$ & $*$ & $*$ & $*$ & $*$ & \\
56 & Rezaei et al. [39] & $*$ & $*$ & $*$ & $*$ & $*$ & $*$ & - & $*$ & $*$ & $*$ & $*$ & $*$ & $*$ & $*$ & $*$ & $*$ & $*$ & $*$ & - & $*$ & $*$ & $*$ & $*$ & $*$ & $*$ & \\
\hline
\end{tabular}

STROBE, Strengthening the Reporting of Observation Studies in Epidemiology; PRISMA, Preferred Reporting Items for Systematic Reviews and Meta-Analyses; CONSORT, Consolidated Standards of Reporting Trials.

cies $(\mathrm{OR}=1.96)$, standing and walking for more than 5 hours during a day in the second trimester were the risk factors for undesirable weight gain during pregnancy $(\mathrm{OR}=1.50)$ [17].

Cetin et al. [18] indicated that pre-eclampsia (PEC) had negative impact on not only pregnancy conditions but also on mental and emotional signs and sleep patterns. Depending on the severity of PEC, these disorders could be more severe. They recommended that obstetric specialists should provide mental health cares and education beside the patients' bed; these services should also be available after labor [18].

\section{Prevalence of Sleep Disorder During Pregnancy and Postpartum}

Prevalence of sleep disorders in the first, second, and third trimesters, and during the whole pregnancy was reported to be $40.1 \%, 53.0 \%, 83.9 \%$, and $59.2 \%$, respectively. Most women (78\%97\%) reported sleep disorders during the third trimester $[6,19]$. In addition, prenatal sleep disorders is associated with a 5.359fold increased risk of postpartum depression during the first 6 weeks postpartum [20]. Gelaye et al. [21] found that those women who were abused during childhood were 1.6 times more affected by sleep disorder due to stress (adjusted odd ratio [AOR] = 1.65), and had 2.11 times poor sleep quality during the first weeks of pregnancy ( $\mathrm{AOR}=2.11$ ). In addition, women who were physically and sexually abused more than twice in their childhood had 2.2 times more sleep disorders because of stress $(\mathrm{AOR}=$ 2.26) and a 2.4- fold poor sleep quality $(\mathrm{AOR}=2.43)$ compared with normal women [21]. According to the International Classification of Sleep Disorders-3 [22], the most prevalent sleep disorders during pregnancy and postpartum with regard to diagnostic criteria are:

\section{Insomnia (disorders of initiating and maintaining sleep, DIMS)}

Insomnia (also known as sleeplessness [23]) and SSD are common in pregnancy. Insomnia is defined as difficulty in falling asleep and typically is followed by daytime sleepiness or sleep duration fewer than 7 hours at night [2]. A study demonstrated an increase in sleeplessness (from $14.3 \%$ in the first trimester to $37.6 \%$ in the third trimester), difficulty in falling asleep (from 14.3 to 44.9 minutes), and reduction in sleep duration (from 8.7 to 7.8 hours) [24].

Prevalence of insomnia in the first, second, and third trimesters of pregnancy and postpartum was $44.2 \%$ (39.3-49.6), 46.3\% (41.9-51.3), 63.7\% (57.7-67.8), and 33.2\% (28.2-37.9), respectively [25]. Risk factors for SSD include nulliparous, obesity, high blood pressure, and old age. These factors are indirectly associated with glucose intolerance during pregnancy [2]. Furthermore, pre-pregnancy insomnia is a risk factor for insomnia in the first trimester of pregnancy ( $\mathrm{AOR}=12.50)$ and obesity is related to insomnia in the third trimester of pregnancy $(\mathrm{AOR}=$ 2.30 ), but moderate physical activity can decrease insomnia in the third trimester $(\mathrm{AOR}=0.65)[25]$.

\section{Sleep related breathing disorders}

Sleep disordered breathing (SDB) is characterized by abnormal respiration and gas exchange during sleep and includes a wide range of signs such as snoring and obstructive sleep apnea (OSA). Snoring in the first trimester is associated with the beginning and continuation of sleepiness and increases depression symptoms in pregnancy [26]. OSA increases from $10.12 \%$ in the first trimester to $31.7 \%$ in the third trimester [24]. OSA is the most common respiratory sleep disorder in women of reproductive age, which is accompanied by side-effects and mortality in pregnant and non-pregnant women [2, 27]. According to the studies, $3.6 \%-8.3 \%$ of the pregnant women complained 
of OSA [19,28,29]. OSA results in continuous hypopnea and apnea, but central sleep apnea is rare in pregnancy [19]. A study showed that the history of gastroesophageal reflux disease in women was $56.7 \%$ and reflux signs had been associated with BMI before pregnancy and labor time, mother's age, smoking, and presence of respiratory sleep disorder signs [30]. Abnormal respiration during sleep (which is mostly, not always, associated with high volume and chronic snore may be due to edema induced by hormonal changes during pregnancy) can be seen in healthy young pregnant women. It may involve the signs of daily sleepiness [31]. The snoring rate increases at the end weeks of pregnancy, but women are less affected by sleep apnea [10]. The rate of snoring has an 18-point increase throughout the pregnancy among women who have a history of snoring [4].

\section{Central disorders of hyper somnolence and circadian rhythm sleep-wake disorders}

Sleep attack or narcolepsy is a common disorder among young women during reproductive age and is characterized by excessive sleepiness during the day, cataplexy, hypnogogic hallucinations, and sleep paralysis. This disorder has a few undesirable consequences because it increases sleep duration [2]. According to the studies, $76 \%$ of the pregnant women have poor sleep quality [12], 38\% have insufficient night sleep (6 hours or less), 52.6\%-65.4\% have daytime sleepiness [26], 61.9\% have sleeplessness, and $100 \%$ have night wake-ups [32]. The rates of disorders increase during pregnancy. $15.2 \%$ of women experience severe daily sleepiness during the whole pregnancy. Nulliparous women and those who snore experience more (2.28-2.1 folds) daily sleepiness during the first trimester. Sleepiness increases in $15.2 \%$ of the women during pregnancy, especially if they have worked for a long time during the week $(\mathrm{OR}=1.04)$, or have had depression signs $(\mathrm{OR}=1.09)$ and snored $(\mathrm{OR}=6.75)$ [26].

\section{Parasomnia}

Parasomnias are undesirable events occurring near sleep onset or within sleep. Parasomnia rate in pregnancy reduces in nulliparous women compared with multiparous women. Talking and walking in sleep decreases in the second trimester compared with before pregnancy (22.8\% vs. $12.6 \%)$. Moreover, sleep onset reduces in the first trimester in comparison with before pregnancy (78.5\% vs. $63.1 \%$ ), but it does not change in the second and third trimesters. 55.7\% of the women reported nightmares from three months before pregnancy to the first $(47.7 \%)$, second (49.5\%), third trimesters (41.2\%), and after labor (40.3\%). Hypnagogic hallucinations decreases in the first trimester compared with before pregnancy ( $6.5 \%$ vs. $9.8 \%$ ), but it returns to the previous level in the next stages of pregnancy. Bruxism rate reduces in the first trimester compared with before pregnancy ( $25.8 \%$ vs. $19.9 \%)$. Although sleep paralysis rate reduces in the first trimester of pregnancy, it is just parasomnia that increases in the next months of pregnancy (from 5.7\% to 13.3\%) [8]. Additionally, undesir- able sleep may result in health-related risks such as metabolism disorders, cardiovascular diseases, and mood disorders $[12,33]$.

Sleep-related movement disorders-restless legs syndrome (RLS) and periodic limb movements in sleep (PLMS)

RLS is a sleep disorder with unknown reasons which leads to an urge for moving legs during the night. It has been observed in more than one-fourth of the pregnant women and has a wide variety and different diagnostic criteria. It is characterized by painful and severe spasm of foreleg muscles resulting in sleep and daily activity disorders [2]. Sleep disorder due to RLS may occur in the forms of increased delay in sleep onset, sleep continuity, and sleeplessness. Studies have demonstrated that 10\%$34 \%$ of the pregnant women have complained of sleeplessness, RLS, and a combination of these problems [28,29]. Gupta et al. [34] suggested that family history, low serum levels of iron and ferritin, high levels of estrogen, vitamin D and calcium deficiency might have an important role in inducing the disease signs. Wilson et al. [35] demonstrated that most RLS patients suffered from PLMS. PLMS not only is associated with sleep disturbance but also may be correlated with autonomic heart rate and arterial hypertension in the third trimester of pregnancy. Despite the role of iron deficiency in RLS; it is not common in PLMS. In addition, $45 \%$ of the women reported more than 5 PLMS and 24\% experienced more than 15 PLMS during the third trimester of pregnancy [35].

\section{Effect of Sleep Disorders on Pregnancy Outcome}

\section{Psychiatric disorders}

At the end stages of pregnancy, symptoms of depression and anxiety are due to sleep disorder, but it is not the case in the midstages [10]. Studies reported that pregnant women with sleep disorder (less than six hours of night sleep and poor sleep quality during the third trimester) were at risk for clinical depression $(23.4 \%-71.7 \%)$ and daytime sleepiness (69\%) [36,37]. Poor sleep quality had been associated with increased depression, perceived stress in pregnancy, psychological distress, and negative consequences on pregnant women and children [7,36,38,39]. Furthermore, prenatal sleep disorders like insomnia symptoms (poor sleep quality, daytime tiredness, short sleep [sleep less than 6 and 7 hours], sleep latency [more than $20 \mathrm{~min}$ ], and sleep loss [more than 2 hours]) were significantly correlated with postnatal depression [40]. Depression before pregnancy was accompanied with pregnancy negative consequences, negative behaviors of children, and long-term psychological consequences in children such as internalizing and externalizing disorders [41]. Volkovich et al. [42] demonstrated that depression and anxiety moderated the relation between objective sleep (with actigraphy for at least five nights) and subjective sleep (based on Pittsburgh Sleep Quality Index). Psychological stresses during pregnancy not only disturb the objective sleep but also subjective sleep [42]. Sleep 
disorders during pregnancy can be the greatest trigger to postpartum psychiatric disorder such as mania, psychosis, depression, and obsessive-compulsive disorder [43].

Also, Sleep deprivation during pregnancy (for $6 \mathrm{~h}$ a day) not only increases the risk for maternal psychological disorders but also leads to harmful consequences in children including less sexual behavior in male children, increased risky behavior, sexual and fertility problems, and emotional and cognitive disturbances in children [36,41,44-47].

As well as, Women with sleep disorders in the first trimester of pregnancy may experience more eating disorders or binge eating disorder (which is defined as eating quickly and large amounts of food; losing control during the binge; and experiencing shame, distress, or guilt afterward) in the second trimester rather than the third trimester of their pregnancy $(\mathrm{OR}=$ 1.26-1.42). However, dissatisfaction with sleep during the whole pregnancy is accompanied by eating disorders that continue until 18 months after labor (ORs $=1.28-1.47)$ [48].

\section{Prenatal outcome}

Primary information about the effect of circadian rhythm on labor indicated that the spontaneous membrane rupture occurred mainly during night and initiation of the following labor pain was early in the morning. Sleep-wake cycle disturbance results in an imbalance between sympathetic, parasympathetic hormones, and excessive secretion of sympathetic hormones. In addition, sleep deprivation releases TNF- $\alpha$, C-reactive protein, and interleukins 1, 6, 10 causing pro-inflammatory conditions [2]. A study proved that most sleep complaints including SSD and undesirable sleep efficiency in the mid and late pregnancy weeks were associated with elevated and stimulated blood levels of IL-6 [49]. These biological pathways are accompanied with unfavorable pregnancy consequences including increased cesarean section $(\mathrm{AOR}=1.60)$, high blood pressure in pregnancy $(\mathrm{AOR}=2.46)$, $\mathrm{PEC}(\mathrm{AOR}=2.42)$ and preterm labor $(\mathrm{AOR}=$ 1.90) [27]. As the prevalence of PEC, blood pressure disorders, and diabetes during pregnancy were $6 \%, 13.1 \%$, and $4.1 \%$, respectively. With the presence of sleep respiratory disorders, the OR of PEC is estimated to be 1.94 and 1.95 at the beginning and mid-stage of pregnancy, respectively. High blood pressure in pregnancy was 1.46 and 1.73 , and pregnancy diabetes was 3.47 and 2.79 at the beginning and mid-stage of pregnancy, respectively [19]. According to the studies, poor or short-term night sleep in Asian women is accompanied with increased gestational diabetes mellitus (GDM) risk, and abnormal regulation of glucose; these two disorders influences on labor type, length of labor stages, neonates Apgar score, and their birth weight [50,51]. RLS has been associated with some pregnancy effects, including PEC and cesarean section [28,52].

Respiratory sleep disorder has been associated with oxidative stress, autonomic function disorder, inflammation, endothelial injury, and changed hormonal regulation for energy consump- tion [19]. OSA is a risk factor for high blood pressure, diabetes, and several metabolic diseases. SSD has been associated with weight gain, glucose intolerance, premature labor, and low birth weight (LBW). Habitual and repeated snores (> 3 nights per week) are accompanied with increased GDM which is exacerbated by obesity [2]. High blood pressure in pregnancy, PEC, and pregnancy diabetes are dependent on SDB. PEC and OSA have a common pathological relationship, but it is not known which one predicts the other one [47].

Qiu et al. [53] reported that short sleep $(\leq 6 \mathrm{~h})(\mathrm{ORs}=2)$ and long sleep $(\geq 9 \mathrm{~h})(\mathrm{ORs}=2.1)$ were accompanied with a twofold increase in the risk of premature placental abruption. Additionally, the complaint of fatigue due to sleep duration was associated with premature placental abruption $(\mathrm{OR}=2.37)$ [53].

\section{Fetal outcome}

SDB can lead to some fetal side-effects, including intrauterine growth retardation (IUGR), preterm labor, LBW, admission to neonatal intensive care unit, and Apgar score less than seven in the first minute of birth [47,54]. Li et al. [55] showed that prenatal SDB didn't significantly relate to Apgar score and birth weight but it was associated with GDM, pregnancy-induced hypertension, and PEC.

Habitual snoring (26\% with initiation of pregnancy and 9\% chronic snoring) is associated with small for or gestational age $(\mathrm{SGA})(\mathrm{OR}=1.65)$ and selective cesarean section $(\mathrm{OR}=2.25)$, but snoring at the beginning of pregnancy $(\mathrm{OR}=1.68)$ has been accompanied with urgent cesarean section [45].

Also, Howe et al. [56] showed that apnea three nights a week or more at the late stages of pregnancy had been associated with SGA $(\mathrm{OR}=2.8)$ and large for gestational age $(\mathrm{LGA})(\mathrm{OR}=2.0)$ but had a stronger correlation with LGA especially when apnea occurred at the initiation of pregnancy. The relationship between apnea and fetus size (especially LGA) was partly due to the relationship between OSA, glucose intolerance, pregnancy diabetes, and macrosomia. Moreover, long sleep duration $(\geq 9 \mathrm{~h}) \mathrm{a}$ week $(\mathrm{OR}=1.6)$ and nasal congestion has been associated with SGA. Findings showed an association between apnea and sudden foreleg spasm at the late stages of pregnancy and neonate consequences. In addition, racial inequalities are also involved in these sleep disorders. Maori women (New Zealand tribes) had higher prevalence of sleep disorders, short sleep length, and poor sleep during pregnancy. Furthermore, RLS $(\mathrm{OR}=3.3)$ and repeated sleep disorders $(\mathrm{OR}=1.7)$ at early pregnancy have been due to excessive hot and cold feelings, accompanied with increased fetal distress risk [56].

Warland et al. [57] emphasized that maternal sleep disorders could predict some poor fetal outcome such as LBW, inappropriate birth growth (LGA, SGA), preterm birth, and stillbirth. Gordon et al. [58] demonstrated that supine position was an additional risk for fetal death at the final stages of pregnancy, especially for women who had been previously at risk. There- 
fore, the sleeping position should be considered in the pregnancies which are suspect of IUGR to reduce fetal death [58].

Toffol et al. [59] demonstrated that clinically depressive pregnant women had SSD, longer sleep latency, higher odds for waking up $[59,60]$. When these disorders continued two or more times during the women night sleep their child associated with all these disorders in the 3.5-year-old [59].

Finally, the results of the present study demonstrated pregnancy could induce some changes in pregnant women sleep such as sleeplessness, repeated wake ups, SSD that could decrease their sleep quality and had sever effects on their pregnancy if we ignored them. Also, sleep disorders not only affected mother (e.g. preterm labor, hypertension, preeclampsia, diabetes, and depression) and fetal outcomes (e.g. IUGR, LGA, SGA, and LBW) but also they could be greatest trigger to postpartum psychiatric disorders (such as mania and psychosis) and harmful sequences during childish period of mother's fetus (e.g. sexual, fertility, emotional, and cognitive problems).

\section{Conclusion}

Findings extracted from the studies enhance the richness of literature about the mutual effects of sleep disorder and pregnancy. Studies also emphasized that the interventional studies should not only be designed for improving sleep during pregnancy but also they should focus on evaluating and managing depression that could be effectively reduced by sleep improvement interventions. Health care providers should assess and improve mothers' sleep health because they have a critical role in the family and are a vulnerable population in the society.

\section{Supplementary Materials}

The online-only Data Supplement is available with this article at https://doi.org/10.17241/smr.2021.00983.

\section{Availability of Data and Material}

All data generated or analyzed during the study are included in this published article (and its supplementary information files).

\section{Author Contributions}

Conceptualization: Rezaei E. Data curation: Rezaei E. Formal analysis: Rezaei E. Funding acquisition: Rezaei E. Investigation: Rezaei E. Methodology: Rezaei E. Project administration: Moghadam ZB. Resources: Rezaei E. Software: Rezaei E. Supervision: Moghadam ZB. Validation: Rahmani A. Visualization: Rahmani A. Writing_original draft: Rezaei E. Writing—review \& editing: all authors.

\section{Conflicts of Interest}

The authors have no potential conflicts of interest to disclose.

\section{Funding Statement}

None.

\section{REFERENCES}

1. Lee KA, Zaffke ME, McEnany G. Parity and sleep patterns during and after pregnancy. Obstet Gynecol 2000;95:14-8.

2. Kumar KV. Sleep disorders in pregnancy: glycaemic implications. $J$
Pak Med Assoc 2016;66(9 Suppl 1):S60-4.

3. Neau JP, Texier B, Ingrand P. Sleep and vigilance disorders in pregnancy. Eur Neurol 2009;62:23-9.

4. Crowley SK, O’Buckley TK, Schiller CE, Stuebe A, Morrow AL, Girdler SS. Blunted neuroactive steroid and HPA axis responses to stress are associated with reduced sleep quality and negative affect in pregnancy: a pilot study. Psychopharmacology (Berl) 2016;233:1299-310.

5. Sut HK, Asci O, Topac N. Sleep quality and health-related quality of life in pregnancy. J Perinat Neonatal Nurs 2016;30:302-9.

6. Effati-Daryani F, Mohammadi A, Zarei S, Mirghafourvand M. Evaluation of sleep quality and its socio-demographic predictors in three trimesters of pregnancy among women referring to health centers in Tabriz, Iran: a cross-sectional study. Evidence Based Care 2019;9:69-76.

7. Jahdi F, Rezaei E, Behboodi MZ, Hagani H. Prevalence of sleep disorders in the pregnant women. PAYESH 2013;12:629-35.

8. Hedman C, Pohjasvaara T, Tolonen U, Salmivaara A, Myllylä VV. Parasomnias decline during pregnancy. Acta Neurol Scand 2002;105:209-14.

9. Centre for Reviews and Dissemination, University of York. Systematic Reviews: CRD's guidance for undertaking reviews in health care [cited 2018 Mar 1]. Available from: https://www.york.ac.uk/media/crd/Systematic_Reviews.pdf.

10. Polo-Kantola P, Aukia L, Karlsson H, Karlsson L, Paavonen EJ. Sleep quality during pregnancy: associations with depressive and anxiety symptoms. Acta Obstet Gynecol Scand 2017;96:198-206.

11. Li G, Kong L, Zhou H, Kang X, Fang Y, Li P. Relationship between prenatal maternal stress and sleep quality in Chinese pregnant women: the mediation effect of resilience. Sleep Med 2016;25:8-12.

12. Felder JN, Laraia B, Coleman-Phox K, Bush N, Suresh M, Thomas M, et al. Poor sleep quality, psychological distress, and the buffering effect of mindfulness training during pregnancy. Behav Sleep Med 2018;16: 611-24.

13. Juulia Paavonen E, Saarenpää-Heikkilä O, Pölkki P, Kylliäinen A, Porkka-Heiskanen T, Paunio T. Maternal and paternal sleep during pregnancy in the child-sleep birth cohort. Sleep Med 2017;29:47-56.

14. Hall WA, Hauck YL, Carty EM, Hutton EK, Fenwick J, Stoll K. Childbirth fear, anxiety, fatigue, and sleep deprivation in pregnant women. $J$ Obstet Gynecol Neonatal Nurs 2009;38:567-76.

15. Gay CL, Richoux SE, Beebe KR, Lee KA. Sleep disruption and duration in late pregnancy is associated with excess gestational weight gain among overweight and obese women. Birth 2017;44:173-80.

16. Abeysena C, Jayawardana P. Sleep deprivation, physical activity and low income are risk factors for inadequate weight gain during pregnancy: a cohort study. J Obstet Gynaecol Res 2011;37:734-40.

17. Moghadam ZB, Rezaei E, Hagani H. The association of fetal sex and common complaints of pregnancy in pregnanat women with sleep disorder. Int J Reprod Biomed (Yazd) 2014;12:122.

18. Cetin O, Guzel Ozdemir P, Kurdoglu Z, Sahin HG. Investigation of maternal psychopathological symptoms, dream anxiety and insomnia in preeclampsia. J Matern Fetal Neonatal Med 2017;30:2510-5.

19. Facco FL, Parker CB, Reddy UM, Silver RM, Koch MA, Louis JM, et al. Association between sleep-disordered breathing and hypertensive disorders of pregnancy and gestational diabetes mellitus. Obstet Gynecol 2017;129:31-41.

20. Chung TC, Chung $\mathrm{CH}$, Peng HJ, Tsao CH, Chien WC, Sun HF. An analysis of whether sleep disorder will result in postpartum depression. Oncotarget 2018;9:25304-14.

21. Gelaye B, Kajeepeta S, Zhong QY, Borba CP, Rondon MB, Sánchez SE, et al. Childhood abuse is associated with stress-related sleep disturbance and poor sleep quality in pregnancy. Sleep Med 2015;16:1274-80.

22. Sateia MJ. International classification of sleep disorders-third edition: highlights and modifications. Chest 2014;146:1387-94.

23. National Institutes of Health. What is insomnia? [cited 2021 May 20]. Available from: https://web.archive.org/web/20160728012148/http:// www.nhlbi.nih.gov/health/health-topics/topics/inso.

24. Madaeva IM, Kolesnikova LI, Protopopova NV, Sakh'ianova NL, Ber- 
dina ON. Features of the sleep pattern during pregnancy. Vestn Ross Akad Med Nauk 2014;69:93-7.

25. Román-Gálvez RM, Amezcua-Prieto C, Salcedo-Bellido I, MartínezGaliano JM, Khan KS, Bueno-Cavanillas A. Factors associated with insomnia in pregnancy: a prospective cohort study. Eur J Obstet Gynecol Reprod Biol 2018;221:70-5.

26. Tsai SY, Lee PL, Lin JW, Lee CN. Persistent and new-onset daytime sleepiness in pregnant women: a prospective observational cohort study. Int J Nurs Stud 2017;66:1-6.

27. Spence DL, Allen RC, Lutgendorf MA, Gary VR, Richard JD, Gonzalez SC. Association of obstructive sleep apnea with adverse pregnancyrelated outcomes in military hospitals. Eur J Obstet Gynecol Reprod Biol 2017;210:166-72.

28. Uglane MT, Westad S, Backe B. Restless legs syndrome in pregnancy is a frequent disorder with a good prognosis. Acta Obstet Gynecol Scand 2011;90:1046-8

29. Minar M, Habanova H, Rusnak I, Planck K, Valkovic P. Prevalence and impact of restless legs syndrome in pregnancy. Neuro Endocrinol Lett 2013;34:366-71.

30. Habr F, Raker C, Lin CL, Zouein E, Bourjeily G. Predictors of gastroesophageal reflux symptoms in pregnant women screened for sleep disordered breathing: a secondary analysis. Clin Res Hepatol Gastroenterol 2013;37:93-9.

31. Guilleminault C, Querra-Salva M, Chowdhuri S, Poyares D. Normal pregnancy, daytime sleeping, snoring and blood pressure. Sleep Med 2000;1:289-97

32. Mindell JA, Cook RA, Nikolovski J. Sleep patterns and sleep disturbances across pregnancy. Sleep Med 2015;16:483-8.

33. St-Onge MP, Grandner MA, Brown D, Conroy MB, Jean-Louis G, Coons $\mathrm{M}$, et al. Sleep duration and quality: impact on lifestyle behaviors and cardiometabolic health: a scientific statement from the American Heart Association. Circulation 2016;134:e367-86.

34. Gupta R, Dhyani M, Kendzerska T, Pandi-Perumal SR, BaHammam AS, Srivanitchapoom P, et al. Restless legs syndrome and pregnancy: prevalence, possible pathophysiological mechanisms and treatment. Acta Neurol Scand 2016;133:320-9.

35. Wilson DL, Walker SP, Fung AM, O'Donoghue FJ, Barnes M, Howard ME. Periodic limb movements in sleep during pregnancy: a common but benign disorder? Sleep Biol Rhythms 2018;16:11-20.

36. Zahra BM, Elham R. The prevalence of depression in pregnant women with sleep disorder. J Psychiatry 2014;17:1000157.

37. Tsai SY, Lin JW, Wu WW, Lee CN, Lee PL. Sleep disturbances and symptoms of depression and daytime sleepiness in pregnant women. Birth 2016;43:176-83.

38. Rezaei E, Moghadam ZB, Saraylu K. Quality of life in pregnant women with sleep disorder. J Family Reprod Health 2013;7:87-93.

39. Rezaei E, Behboodi Moghadam Z, Hagani H. The effect of sleep health behavioral education on the depression of pregnant women with sleep disorders: a randomized control trial. Iran Red Crescent Med J 2015;17: e11420.

40. Pietikäinen JT, Polo-Kantola P, Pölkki P, Saarenpää-Heikkilä O, Paunio T, Paavonen EJ. Sleeping problems during pregnancy-a risk factor for postnatal depressiveness. Arch Womens Ment Health 2019;22:327-37.

41. Peng Y, Wang W, Tan T, He W, Dong Z, Wang YT, et al. Maternal sleep deprivation at different stages of pregnancy impairs the emotional and cognitive functions, and suppresses hippocampal long-term potentiation in the offspring rats. Mol Brain 2016;9:17.

42. Volkovich E, Tikotzky L, Manber R. Objective and subjective sleep during pregnancy: links with depressive and anxiety symptoms. Arch
Womens Ment Health 2016;19:173-81.

43. Sharma V. Role of sleep deprivation in the causation of postpartum obsessive-compulsive disorder. Med Hypotheses 2019;122:58-61.

44. Alvarenga TA, Aguiar MF, Mazaro-Costa R, Tufik S, Andersen ML. Effects of sleep deprivation during pregnancy on the reproductive capability of the offspring. Fertil Steril 2013;100:1752-7.

45. O'Brien LM, Bullough AS, Owusu JT, Tremblay KA, Brincat CA, Chames MC, et al. Snoring during pregnancy and delivery outcomes: a cohort study. Sleep 2013;36:1625-32.

46. Yari F, Moghadam ZB, Parvizi S, Nayeri ND, Rezaei E. Sexual and reproductive health problems of female university students in Iran: a qualitative study. Glob J Health Sci 2015;7:278-85.

47. Carnelio S, Morton A, McIntyre HD. Sleep disordered breathing in pregnancy: the maternal and fetal implications. J Obstet Gynaecol 2017; 37:170-8.

48. Ulman TF, Von Holle A, Torgersen L, Stoltenberg C, Reichborn-Kjennerud T, Bulik CM. Sleep disturbances and binge eating disorder symptoms during and after pregnancy. Sleep 2012;35:1403-11.

49. Stein A, Pearson RM, Goodman SH, Rapa E, Rahman A, McCallum $\mathrm{M}$, et al. Effects of perinatal mental disorders on the fetus and child. Lancet 2014;384:1800-19.

50. Zafarghandi N, Hadavand S, Davati A, Mohseni SM, Kimiaiimoghadam F, Torkestani F. The effects of sleep quality and duration in late pregnancy on labor and fetal outcome. J Matern Fetal Neonatal Med 2012;25:535-7.

51. Cai S, Tan S, Gluckman PD, Godfrey KM, Saw SM, Teoh OH, et al. Sleep quality and nocturnal sleep duration in pregnancy and risk of gestational diabetes mellitus. Sleep 2017;40:zsw058.

52. Minar M, Habanova H, Rusnak I, Planck K, Valkovic P. Prevalence and impact of restless legs syndrome in pregnancy. Neuro Endocrinol Lett 2013;34:36-71.

53. Qiu C, Sanchez SE, Gelaye B, Enquobahrie DA, Ananth CV, Williams MA. Maternal sleep duration and complaints of vital exhaustion during pregnancy is associated with placental abruption. J Matern Fetal Neonatal Med 2015;28:350-5.

54. Bourjeily G, Danilack VA, Bublitz MH, Muri J, Rosene-Montella K, Lipkind $\mathrm{H}$. Maternal obstructive sleep apnea and neonatal birth outcomes in a population based sample. Sleep Med 2020;66:233-40.

55. Li L, Zhao K, Hua J, Li S. Association between sleep-disordered breathing during pregnancy and maternal and fetal outcomes: an updated systematic review and meta-analysis. Front Neurol 2018;9:91.

56. Howe LD, Signal TL, Paine SJ, Sweeney B, Priston M, Muller D, et al. Self-reported sleep in late pregnancy in relation to birth size and fetal distress: the E Moe, Māmā prospective cohort study. BMJ Open 2015;5: e008910.

57. Warland J, Dorrian J, Morrison JL, O’Brien LM. Maternal sleep during pregnancy and poor fetal outcomes: a scoping review of the literature with meta-analysis. Sleep Med Rev 2018;41:197-219.

58. Gordon A, Raynes-Greenow C, Bond D, Morris J, Rawlinson W, Jeffery H. Sleep position, fetal growth restriction, and late-pregnancy stillbirth: the Sydney stillbirth study. Obstet Gynecol 2015;125:347-55.

59. Toffol E, Lahti-Pulkkinen M, Lahti J, Lipsanen J, Heinonen K, Pesonen $\mathrm{AK}$, et al. Maternal depressive symptoms during and after pregnancy are associated with poorer sleep quantity and quality and sleep disorders in 3.5-year-old offspring. Sleep Med 2019;56:201-10.

60. Rezaei E, Moghadam Behboodi Z. The association of depression and common complaints of pregnancy in pregnanat women with sleep disorder. Iranian Journal of Reproductive Medicine 2014;12(6) Suppl:122. 
(((“Pregnant Women”[Mesh] OR “Pregnancy”[Mesh]) OR ( "Pregnancy Trimester, Third”[Mesh] OR “Pregnancy Trimester, Second”[Mesh]

OR “Pregnancy Trimester, First”[Mesh] )) OR ( "Postpartum Period”[Mesh] OR "Postnatal Care”[Mesh] )) AND “Sleep Wake

Disorders"[Mesh]

(((“Pregnant Women”[Mesh] OR “Pregnancy”[Mesh]) OR ( "Pregnancy Trimester, Third”[Mesh] OR “Pregnancy Trimester, Second”[Mesh] OR “Pregnancy Trimester, First”[Mesh] )) OR ( “Postpartum Period”[Mesh] OR “Postnatal Care”[Mesh] )) AND “Dyssomnias”[Mesh]

(((“Pregnant Women”[Mesh] OR “Pregnancy”[Mesh]) OR ( "Pregnancy Trimester, Third”[Mesh] OR “Pregnancy Trimester, Second”[Mesh] OR "Pregnancy Trimester, First”[Mesh] )) OR ( "Postpartum Period”[Mesh] OR “Postnatal Care”[Mesh] )) AND “Sleep Disorders, Circadian Rhythm”[Mesh]

(((“Pregnant Women”[Mesh] OR “Pregnancy”[Mesh]) OR ( "Pregnancy Trimester, Third”[Mesh] OR “Pregnancy Trimester, Second”[Mesh] OR “Pregnancy Trimester, First”[Mesh] )) OR ( "Postpartum Period”[Mesh] OR "Postnatal Care”[Mesh] )) AND “Sleep Deprivation"[Mesh]

(((“Pregnant Women”[Mesh] OR “Pregnancy”[Mesh]) OR ( "Pregnancy Trimester, Third”[Mesh] OR “Pregnancy Trimester, Second”[Mesh] OR “Pregnancy Trimester, First”[Mesh] )) OR ( “Postpartum Period”[Mesh] OR “Postnatal Care”[Mesh] )) AND “Parasomnias”[Mesh]

(((“Pregnant Women”[Mesh] OR “Pregnancy”[Mesh]) OR ( "Pregnancy Trimester, Third”[Mesh] OR “Pregnancy Trimester, Second”[Mesh] OR “Pregnancy Trimester, First”[Mesh] )) OR ( "Postpartum Period”[Mesh] OR "Postnatal Care”[Mesh] )) AND “Restless Legs Syndrome"[Mesh]

(((“Pregnant Women”[Mesh] OR “Pregnancy”[Mesh]) OR ( "Pregnancy Trimester, Third”[Mesh] OR “Pregnancy Trimester, Second”[Mesh] OR “Pregnancy Trimester, First”[Mesh] )) OR ( "Postpartum Period”[Mesh] OR "Postnatal Care”[Mesh] )) AND “Sleep Arousal Disorders"[Mesh]

(((“Pregnant Women”[Mesh] OR “Pregnancy”[Mesh]) OR ( "Pregnancy Trimester, Third”[Mesh] OR “Pregnancy Trimester, Second”[Mesh] OR “Pregnancy Trimester, First”[Mesh] )) OR ( "Postpartum Period”[Mesh] OR "Postnatal Care”[Mesh] )) AND (“Sleep Wake Disorders"[Mesh] OR “Dyssomnias”[Mesh] OR “Sleep Disorders, Circadian Rhythm”[Mesh] OR “Sleep Deprivation”[Mesh] OR "Parasomnias"[Mesh] OR "Restless Legs Syndrome”[Mesh] OR “Sleep Arousal Disorders”[Mesh])) 